\title{
Electrical properties of silver selenide thin films prepared by reactive evaporation
}

\author{
M C SANTHOSH KUMAR and B PRADEEP* \\ Department of Physics, Cochin University of Science and Technology, Cochin 682 022, India
}

MS received 29 July 2001; revised 17 August 2002

\begin{abstract}
The electrical properties of silver selenide thin films prepared by reactive evaporation have been studied. Samples show a polymorphic phase transition at a temperature of $403 \pm 2 \mathrm{~K}$. Hall effect study shows that it has a mobility of $2000 \mathrm{~cm}^{2} \mathrm{~V}^{-1} \mathrm{~s}^{-1}$ and carrier concentration of $10^{18} \mathrm{~cm}^{-3}$ at room temperature. The carriers are of $n$-type. $X$-ray diffraction study indicates that the as-prepared films are polycrystalline in nature. The lattice parameters were found to be $a=4.353 \AA, b=6.929 \AA$ and $c=7.805 \AA$.
\end{abstract}

Keywords. Thin film; silver selenide; reactive evaporation; electrical conductivity.

\section{Introduction}

Silver selenide attracts the interest of researchers because of its application in the switching devices. The binary and ternary semiconductors are the basic materials for schottky barriers, solar cells and electronic engineering. Moreover, silver selenide belongs to the family of superionic conductors, which are of present and future technological interest. It is a well known fact that silver selenide undergoes a polymorphic phase transition. Many researchers have reported different values for this transition temperature. Damodara Das and Karunakaran (1989, 1990) reported the thickness dependence of transition temperature and activation energy. The low temperature phase below $400 \mathrm{~K}$ was identified as $\beta-\mathrm{Ag}_{2} \mathrm{Se}$ with a structure of orthorhombic and the high temperature $\alpha$ $\mathrm{Ag}_{2} \mathrm{Se}$ has bcc structure. According to Abdullayev et al (1983), silver selenide exists as a high temperature cubic phase $(\alpha)$ and low temperature orthorhombic $(\beta)$ and tetragonal phase with transition temperature of about $408 \mathrm{~K}$ or $395 \mathrm{~K}$. Moreover, the $\beta$ to $\alpha$ transition does not occur directly but through an intermediate phase; the duration of this new phase increases from cycle to cycle. The structural data available in literature are contrary for the low temperature phase. The compound has been described as tetragonal, orthorhombic and monoclinic by different authors. Ridder and Amelinckx (1973) reviewed different structural parameters reported by different researchers. This may be due to the differences in the preparation technique.

Many methods have been used for the preparation of silver selenide thin films. Damodara Das and Karunakaran (1989) prepared the films by vacuum evaporation

\footnotetext{
*Author for correspondence
}

of silver selenide alloy followed by annealing. Abdullayev et al (1983) used the explosive evaporation of the powdered compound in vacuum on different substrates. Okabe and Ura (1994) prepared films by flash evaporation. Sharma et al (1990) reported chemical bath deposition technique. Somogyi and Safran (1995a,b) and Safran et al (1992) adopted a method to deposit silver from a molybdenum boat on to substrates at $120^{\circ} \mathrm{C}$ and sublimated selenium over it at a substrate temperature of $200^{\circ} \mathrm{C}$ at a rate of 0.05 to $0.1 \mathrm{~nm} \mathrm{~s}^{-1}$. Kulkarni et al (1995) had reported the conversion of silver films to silver selenide films by chemical method. Bernede et al (1982) used a method of evaporating powdered silver selenide from a tantalum boat. But it undergoes partial decomposition. The films show metallic behaviour between $77 \mathrm{~K}$ and $300 \mathrm{~K}$ and with a chemical composition, $\mathrm{Ag}_{2-x} \mathrm{Se}_{1+x}$ with $0.01 \leq x \leq 0 \cdot 05$.

Kaito et al (1982) used a method of vacuum deposition of selenium on glass substrates cooled to liquid nitrogen temperature. Holey silver films were prepared by vacuum evaporation on cellulose acetobutyrate. The selenium films were wet stripped from the substrate and mounted on the holey silver films. $\mathrm{Ag}_{2} \mathrm{Se}$ crystals were produced by reaction of selenium films with silver films at room temperature. Constantinescu (1976) investigated the properties of thin films prepared by separate evaporation of components ( $\mathrm{Ag}$ first and then $\mathrm{Se}$ ) in a vacuum of about $10^{-6} \mathrm{~m} \cdot$ bar on $\mathrm{NaCl}$ and $\mathrm{KCl}$ substrates. Saito et al (1981) also reported the same technique. This variety in the preparation technique leads to composition inconsistency. In this context we tried a method in which not much work has been reported. We report here preparation of the $\mathrm{Ag}_{2} \mathrm{Se}$ thin film by reactive evaporation and its electrical conductivity, Hall coefficient and mobility. 


\section{Experimental}

In the present work we report the reactive evaporation of silver selenide thin films. This technique is also known as Gunther's technique or three-temperature method (Anderson 1966). It is based on the fact that continuous condensation of a given vapour at a given rate takes place only if the temperature of the substrate drops below a certain critical value. For many binary systems, it has been found that a stoichiometric interval exists with limited degree of freedom in selecting the individual components and substrate temperature for obtaining a particular compound of the system (George and Pradeep 1985; John et al 1994). In this work we employed a conventional vacuum system with two separate sources. The silver atoms reaching the substrate react with selenium atoms present on the substrate surface, and the compound film is formed. The need of synthesis of compound from elements prior to deposition, which is a tedious process, can be eliminated by this technique. Moreover, the requirement of high temperature for evaporation of compound and subsequent decomposition are eliminated. Because of the high volatile elemental pressure, some unreacted volatile element is likely to be trapped in the growing film, changing the film properties, especially at low substrate temperature is the disadvantage of this technique.

Cleaned optically flat glass substrates were used for the preparation. High purity silver $(99.999 \%)$ and selenium $(99.99 \%)$ were utilized for evaporation. Silver was evaporated from a molybdenum boat and selenium from a glass crucible kept in a molybdenum basket. The glass substrate was mounted on a substrate holder with a heating arrangement and temperature was measured with the help of a fine wire chromel and alumel thermocouple. The temperature of the substrate was maintained at $398 \pm 5 \mathrm{~K}$ throughout the deposition. The rate of deposition and impingement rate were calculated for different components. The impingement rate is given by the equation

$$
d N_{\mathrm{m}} / A_{\mathrm{r}} d t=N_{\mathrm{a}} \rho_{\mathrm{m}} d^{\prime} / M_{\mathrm{m}},
$$

where $N_{\mathrm{a}}$ is the Avogadro number, $\rho_{\mathrm{m}}$ the density of the bulk material in $\mathrm{g} \mathrm{cm}^{-3}, M_{\mathrm{m}}$ the molar mass of the material in $\mathrm{g} \mathrm{mol}^{-1}, A_{\mathrm{r}}$ the reactive surface area in $\mathrm{cm}^{2}$ and $d^{\prime}$ the deposition rate in $\mathrm{cm} \mathrm{s}^{-1}$. The following deposition parameters were used for obtaining silver selenide thin films.

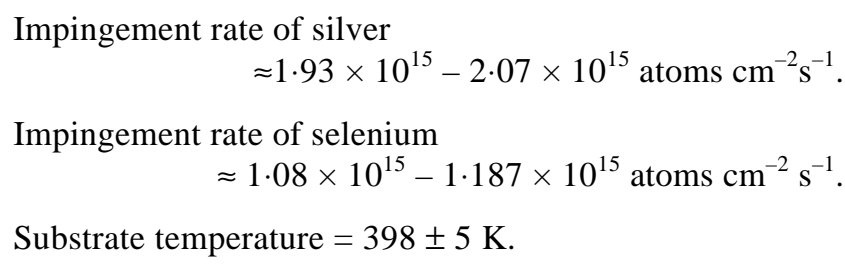

The deposition rate was controlled by adjusting the current through the filaments. A vacuum of the order of
$10^{-5} \mathrm{~m} \cdot$ bar was maintained in the chamber throughout the deposition. The substrate temperature had been maintained at the coating temperature for $45 \mathrm{~min}$ to $1 \mathrm{~h}$ after deposition to stabilize the reactions.

Electrical conductivity and Hall effect measurements were carried out on the as prepared samples. Film thickness were measured using the well known Tolansky's multiple beam interferometric technique (Tolansky 1948).

\section{Results and discussion}

The most accepted crystal structure of low temperature $\mathrm{Ag}_{2} \mathrm{Se}$ is orthorhombic with the $P 2_{\mathrm{I}} 2_{\mathrm{I}} 2_{\mathrm{I}}$ space group. Figure 1 shows the X-ray diffraction pattern of a typical silver selenide thin film prepared at a substrate temperature of $398 \pm 5 \mathrm{~K}$. This indicates the existence of (002), (013), (004) and (014) planes of reflections of orthorhombic phase of silver selenide. When annealed no additional peak was found. Table 1 shows the $d$ spacing observed along with the standard data (ASTM card 241041). The lattice parameters were calculated and its values are $a=4.353 \AA, b=6.929 \AA$ and $c=7.805 \AA$, which are very close to the reported values. Damodara Das and Karunakaran (1989) reported the XRD pattern showing only (002) and (004) planes. Kulkarni et al (1995) obtained a film of orientations (002), (111), (210) and (204) planes. The grain size of the as prepared films has been calculated using the Scherrer formula. It is found that the grain size is of the order of $36 \mathrm{~nm}$.

The electrical characterization was carried out in a conductivity set-up coupled with a rotary pump to pump down to $10^{-3} \mathrm{~m} \cdot$ bar. Samples with dimension $2.5 \times 30 \mathrm{~mm}$ were used for the study. A constant voltage was applied across the sample and the variation of current with temperature was studied using silver paste as the ohmic contact. Figure 2 shows the variation of conductivity $(\sigma)$ with temperature of the sample for four continuous heating and cooling cycles. It shows the existence of hysteresis in the conductivity with temperature. Films showed a

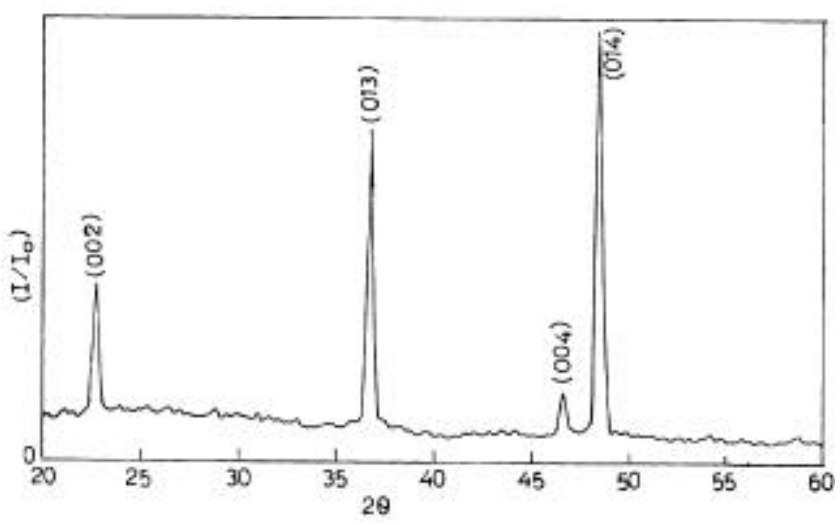

Figure 1. X-ray diffractogram of the silver selenide thin film prepared at $398 \mathrm{~K}$. 
semiconducting nature up to $403 \mathrm{~K}$ and then it showed a metallic nature with rise in temperature. The polymorphic phase transition temperature was observed at $403 \pm 2 \mathrm{~K}$ for every sample. The reverse transition occurred at about $373 \pm 5 \mathrm{~K}$ while cooling. This shows the reversible nature of the transition. The transition temperature was observed to decrease to $390 \pm 2 \mathrm{~K}$ for the second cycle of heating. From the figure it is clear that after the second cycle of heating and cooling the electrical properties remain the same i.e. the conductivity variation with temperature retraces nearly the same path of second cycle. Abdullayev et al (1983) studied the phase transition phenomenon using the kinematic electron diffraction technique. Analysis of this pattern revealed that heating results in the conversion of the initial $\beta-\mathrm{Ag}_{2} \mathrm{Se}$ to $\alpha$ $\mathrm{Ag}_{2} \mathrm{Se}$ while cooling induces the reverse process. According to them the temperature of the reverse transition from the $\alpha$ to $\beta$ phase was found to increase with repeated thermal cycling; while it was only $343 \mathrm{~K}$ for the first cycle, it had increased to $383 \mathrm{~K}$ by the sixth cycle. In the present case the reverse transition temperature decreased from $378 \mathrm{~K}$ in the first cycle to $367 \mathrm{~K}$ in the second cycle and it was $373 \mathrm{~K}$ in the third and fourth cycles.

The grain size was found to increase from $36 \mathrm{~nm}$ to $43 \mathrm{~nm}$ after the first thermal cycle. These observations

Table 1. X-ray diffraction data for $\mathrm{Ag}_{2} \mathrm{Se}$ thin film prepared at $398 \mathrm{~K}$.

\begin{tabular}{llrlr}
\hline & \multicolumn{2}{c}{ Standard pattern } & \multicolumn{2}{c}{ Prepared film } \\
\hline & (ASTM card: $24-1041)$ & \multicolumn{2}{c}{} \\
\hline$(h k l)$ & $d(\AA)$ & $I / I_{0}$ & $d(\AA)$ & $I / I_{0}$ \\
$(002)$ & $3 \cdot 877$ & 6 & $3 \cdot 9141$ & 36 \\
$(013)$ & $2 \cdot 429$ & 31 & $2 \cdot 4436$ & 66 \\
$(004)$ & $1 \cdot 940$ & 7 & 1.9455 & 13 \\
$(014)$ & $1 \cdot 872$ & 23 & $1 \cdot 8773$ & 100 \\
\hline
\end{tabular}

show that the phase transition process is the result of an increase in the number of crystallization centres, the critical dimension of which can be achieved in a very short time. The increase in electrical conductivity after the first thermal cycle can be expected because of the increase in the grain size and removal of imperfections by heating cooling cycle as evident from the XRD measurement. Phase transition temperature is inversely proportional to the thickness of the film and the bulk material shows much less transition temperature (Damodara Das and Karunakaran 1990). Therefore, the decrease in transition temperature after the first cycle is invariably due to the increase in the grain size. The shift in phase transition temperature, $\Delta T$, can be expressed using the following expression assuming that the grains are spherical

$$
\Delta T=T_{\mathrm{B}}-T_{\mathrm{T}}=\Delta \sigma T_{\mathrm{B}} / L t,
$$

where $L$ is the heat of transformation, $T_{\mathrm{B}}$ the transition temperature in the bulk material and $T_{\mathrm{T}}$ the transition temperature in the thin film state with thickness $t$; $\Delta \sigma=\sigma_{2}-\sigma_{1}$ is the change in the function of specific external surface and grain boundary surface energies during phase transition. $\sigma_{1}$ refers to the high temperature and $\sigma_{2}$ refers to the low temperature phases (Damodara Das and Karunakaran 1990). This expression is valid in our case also as the grain size is a function of thickness and we replace thickness to grain size. $T_{\mathrm{T}}$ can increase or decrease, depending on whether $\Delta \sigma$ is positive or negative. In the phase transition of $\mathrm{Ag}_{2}$ Se thin films, since the transition temperature increases with a decrease of thickness, it can also be inferred that $\Delta \sigma$ is negative.

Silver selenide is a non-stoichiometric compound in both $\alpha$ and $\beta$-phase and dissolved $\mathrm{Ag}$ atoms are present. The phase transition and hysteresis are related to the kinetics of the nucleation of the phase transition of the $\beta$ phase and clustering of Ag atoms at trapping centres (Kumashiro et al 1996). The high temperature phase

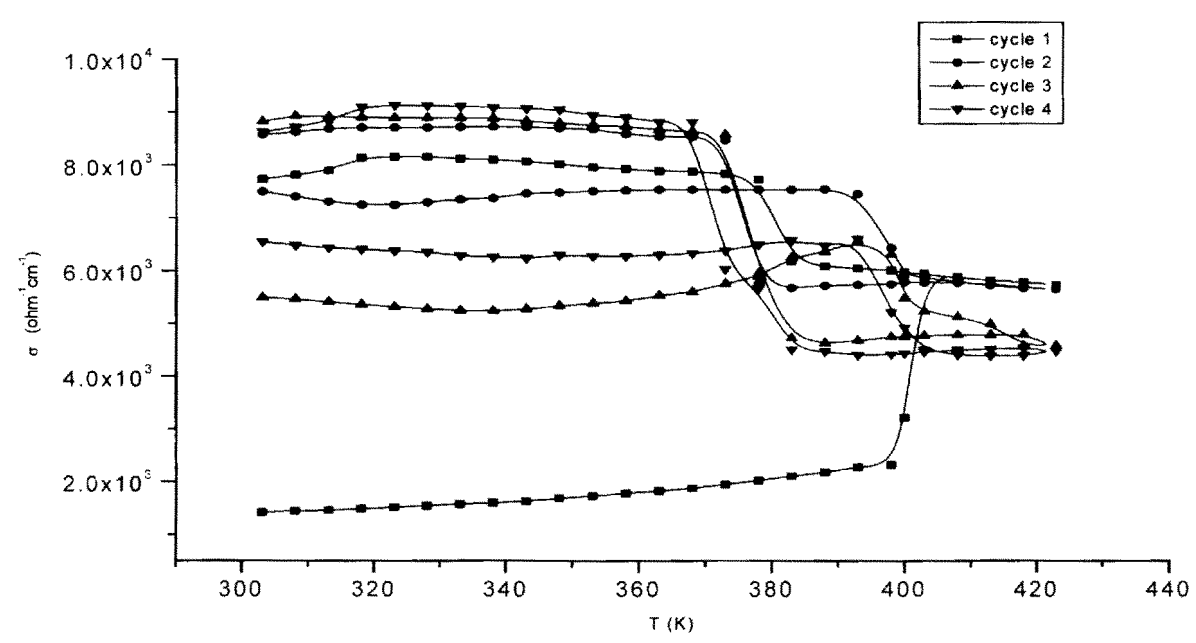

Figure 2. Variation of conductivity $(\sigma)$ with temperature showing four continuous cycles. 
behaves as a metal and its structure is body centred cubic, with lattice parameter $a=0.498 \mathrm{~nm}$ and two $\mathrm{Ag}_{2} \mathrm{Se}$ molecules in a unit cell. The Se and Ag atoms, which are statistically distributed among 42 interstitial sites of the unit cell, preferentially filling up the largest interstices, form the $b c c$ lattice. Such a structure predicts that all the $\mathrm{Ag}$ ions are mobile. Hence ionic conductivity due to $\mathrm{Ag}$ ions is large in the high-temperature phase and increases slightly with increasing temperature.

From the $\ln I$ vs $1 / T$ curve the activation energy was calculated. It was found that activation energy was $0.058 \mathrm{eV}$ for films of thickness of the order of $200 \mathrm{~nm}$. Figure 3 shows the $\ln I$ vs $1 / T$ graph from $300 \mathrm{~K}$ to $400 \mathrm{~K}$. Ferhat and Nagao (2000) studied the electrical properties of $\mathrm{Ag}_{2} \mathrm{Se}$ from $70 \mathrm{~K}$ to $300 \mathrm{~K}$. At room temperature they observed a conductivity of 770, 1988 and $826 \mathrm{ohm}^{-1} \mathrm{~cm}^{-1}$ for their three different samples.
Figure 4 shows the variation of Hall coefficient, $R_{\mathrm{H}}$, with temperature. $R_{\mathrm{H}}$ decreases with increase in temperature. At and around the transition temperature it shows a sudden fall and then remains almost constant. The variation of mobility, $\mu_{\mathrm{H}}$, with temperature is shown in figure 5 . Mobility varies from $2000 \mathrm{~cm}^{2} \mathrm{~V}^{-1} \mathrm{~s}^{-1}$ to $250 \mathrm{~cm}^{2} \mathrm{~V}^{-1} \mathrm{~s}^{-1}$ with temperature. The type of carrier was found to be of electrons. Damodara Das and Karunakaran (1989) reported a value of $2000 \mathrm{~cm}^{2} \mathrm{~V}^{-1} \mathrm{~s}^{-1}$ for mobility at room temperature. Somogyi and Safran (1995a,b) also reported the drastic fall in $\mu_{\mathrm{H}}$ near the transition temperature.

Carrier concentration varies from $10^{18} \mathrm{~cm}^{-3}$ to $10^{19} \mathrm{~cm}^{-3}$ with increase in temperature. At the phase transition temperature it was observed that the carrier concentration suddenly increased by a factor of ten. Somogyi et al (1994) observed irregularities in mobility and Hall coefficient of monocrystalline and polycrystalline

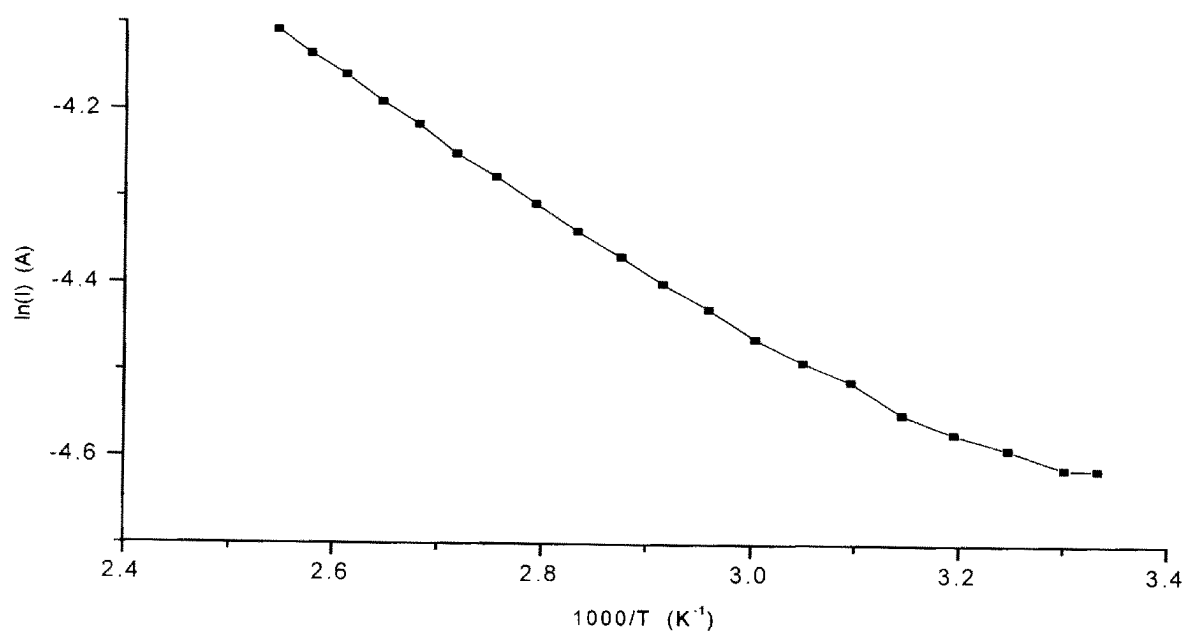

Figure 3. Variation of $\ln (I)$ versus inverse temperature of a typical silver selenide thin film below $400 \mathrm{~K}$.

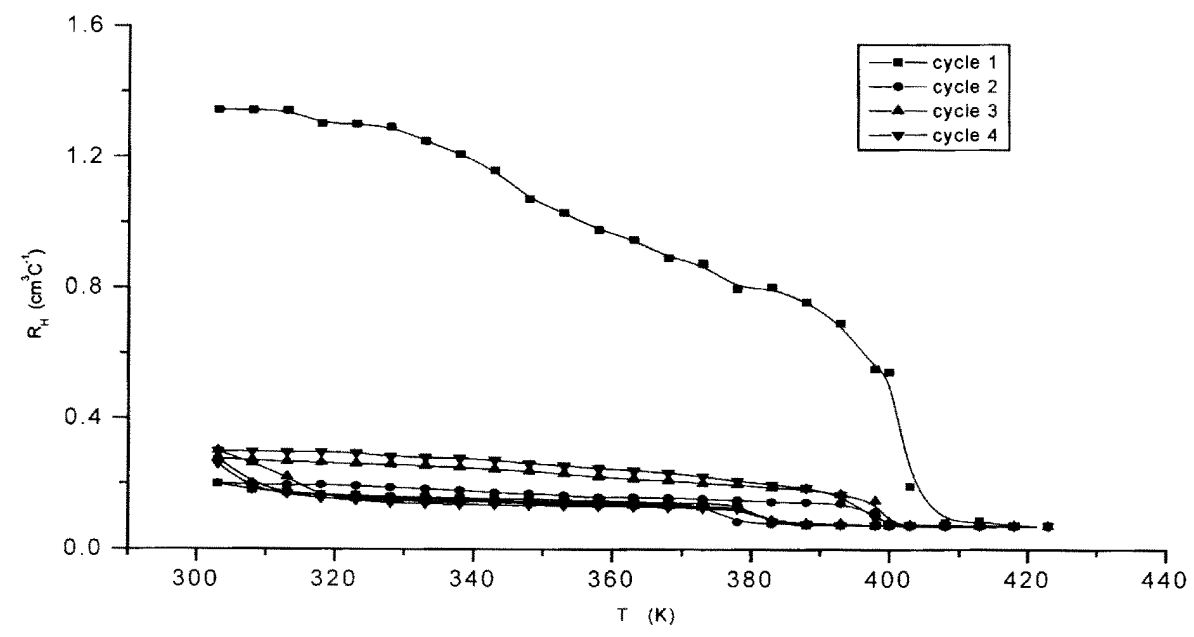

Figure 4. Variation of Hall coefficient $\left(R_{\mathrm{H}}\right)$ with temperature showing four continuous cycles. 


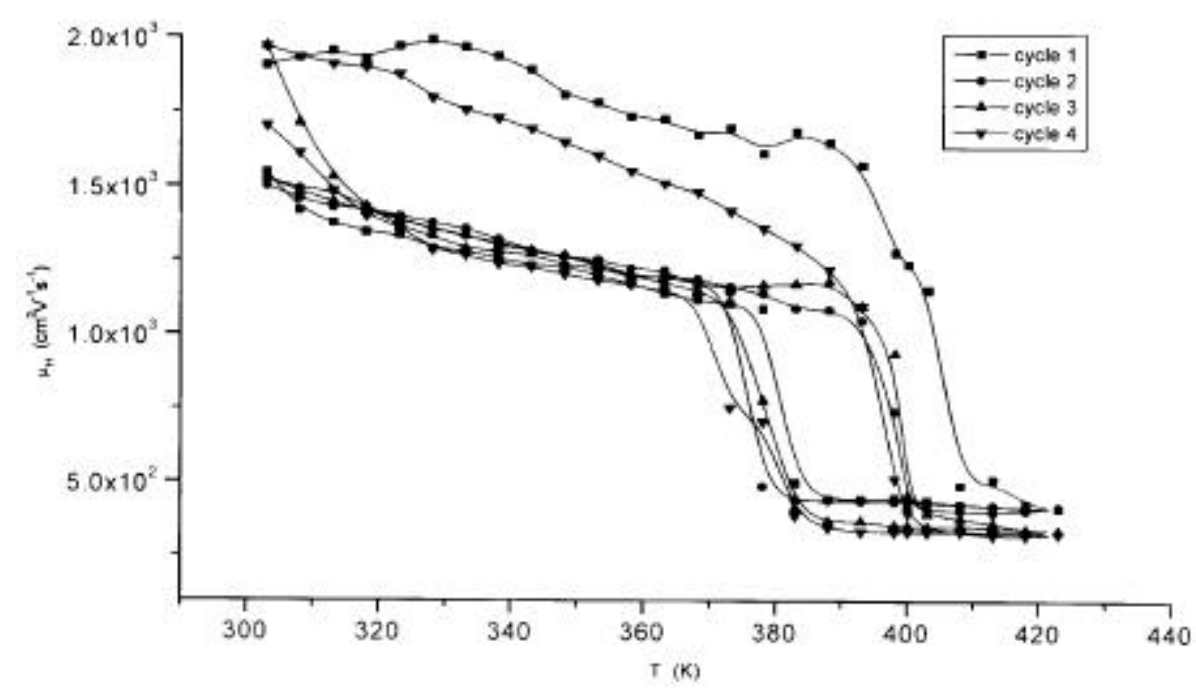

Figure 5. Variation of Hall mobility $(\mu)$ with temperature showing four continuous cycles.

samples of silver selenide. But in the present work we did not observe such irregularities.

\section{Conclusions}

Silver selenide thin films were prepared by reactive evaporation. X-ray studies revealed the formation of silver selenide. Films formed were of polycrystalline nature. Samples showed a semiconducting nature up to $403 \mathrm{~K}$ above which, they showed a metallic behaviour. This may be due to its structural change from orthorhombic to cubic phase. The polymorphic phase transition was observed at $403 \pm 2 \mathrm{~K}$. The decrease in transition temperature is due to the increase of grain size after the thermal cycle. The Hall coefficient, carrier mobility and carrier concentration were measured.

\section{References}

Abdullayev A G, Shafizade R B, Krupnikov E S and Kiriluk K V 1983 Thin Solid Films 106175

Anderson J C 1966 The use of thin films in physical investigations (London: Academic Press) p. 213

Bernede J C, Conan A, Fouesnant E, Bouchairi B El and Goureaux G 1982 Thin Solid Films 97165
Constantinescu L V 1976 Thin Solid Films 32333

Damodara Das V and Karunakaran D 1989 Phys. Rev. B39 10872

Damodara Das V and Karunakaran D 1990 J. Appl. Phys. 68 878

Ferhat Marhoun and Nagao Jiro 2000 J. Appl. Phys. 88813

George J and Pradeep B 1985 Solid State Commun. 56117

John K J, Pradeep B and Mathai E 1994 J. Mater. Sci. 291581

Kaito C, Nakamura N, Teranishi K, Sekimoto S and Shijori M 1982 Phys. Status Solidi (a) 71109

Kulkarni A B, Uplane M D and Lokhande C D 1995 Thin Solid Films 12014

Kumashiro Y, Ohachi T and Taniguchi I 1996 Solid State Ionics $\mathbf{8 6} 761$

Okabe Toshio and Ura Katsuhiko 1994 J. Appl. Cryst. 27140

Ridder R De and Amelinckx S 1973 Phys. Status Solidi (a) 18 99

Safran G, Geszti O, Radnoczi G and Barna P B 1992 Thin Solid Films 215147

Saito Y, Sato M and Shijori M 1981 Thin Solid Films 79257

Sharma K C, Sharma R P and Garg J C 1990 Indian J. Pure \& Appl. Phys. 28546

Somogyi K and Safran G 1995a Vacuum 461055

Somogyi K and Safran G 1995b J. Appl. Phys. 786855

Somogyi K, Panine P and Safran G 1994 Acta Phys. Hung. 74 243

Tolansky S 1948 Multiple beam interferometry of surface and films (London: Oxford University Press) 